\title{
ETIOLOGY OF PERINATAL STROKE; A ROLE FOR PROTHROMBOTIC COAGULATION
} FACTORS?

\author{
A. Spalice ${ }^{1}$, F. Del Balzo ${ }^{2}$, M. Balestri ${ }^{3}$, F. Greco ${ }^{4}$, M. Campelli ${ }^{5}$, M. Ruggieri ${ }^{4}$, M.R. Cilio ${ }^{3}$, P. Colarizi ${ }^{1}$, E. \\ Mercuri $^{6}$ \\ ${ }^{1}$ Dept. of Pediatrics, ${ }^{2}$ Univ La Spienza, ${ }^{3}$ Bambino Gesù Children's Hospital, Roma, ${ }^{4}$ Dept. of Pediatrics, \\ Univ of Catania, Catania, ${ }^{5}$ Dept. of Pediatrics, ${ }^{6}$ Dept. of Pediatrics, Univ Cattholic, Roma, Italy
}

Objective: Stroke is relatively rare in children and the clinical presentation of perinatal stroke is often subtle. In perinatal stroke, multiple, often coexisting, risk factors are involved, varying from maternal and fetal risk factors during pregnancy and delivery, to infectious causes and cardiac diseases as well as medical interventions and congenital prothrombotic coagulation factors.

Methods: To explore the prevalence of different predisposing conditions in perinatal stroke patients we evaluated 96 patients (43 males; 53 females), including subjects with ischemic and hemorrhagic stroke subtypes. Baseline investigations included complete blood count, total cholesterol, triglycerides, lipoprotein (a), prothrombin, activated thromboplastin, plasma fibrinogen level, activity of protein $\mathrm{C}$ sensitivity ratio, total plasma homocysteine, lupus anticoagulant, anticardiolipin, and anti- $\beta 2$ glycoprotein 1 antibodies. DNA analysis was performed for the Factor V Leiden mutation, Factor II G20219A variant, and the thermolabile variant of MTHFR.

Results: The major genetic risk factor in our series of patients was heterozygosity and homozigosity for the MTHFR C677T mutation (39/96 patients; 40\%) in 5 patients was associated with the Factor V Leiden mutation, in 6 with deficiency of activity of protein C. Acquired predisposing conditions were present in 18/96 (18\%) patients and included Threatened abortion, oligohydramnios, intra uterine growth retardation, gestosis, chorioamniositis. In 7 patients both genetic and aquired predisposing factors were present.

Conclusion: Our results emphasize that prothrombotic coagulation risk factors, especially MTHFR mutation, can predispose to perinatal stroke, alone or in combination with other genetic or acquired factors. 